\title{
POTENCIALIDADES DA MANUFATURA ADITIVA PARA CONSTRUÇÃO CIVIL
}

\author{
SOUZA, Hemani Alves Furfuro de \\ Universidade Federal de Viçosa, e-mail: hemani@ufv.br \\ MARTINEZ, Andressa Carmo Pena \\ Universidade Federal de Viçosa, e-mail: andressamartinez@ufv.br \\ TIBÚRCIO, Túlio Márcio de Salles \\ Universidade Federal de Viçosa, e-mail: tmst83@hotmail.com
}

\begin{abstract}
RESUMO
O objetivo geral desta pesquisa de mestrado baseia-se na compreensão, análise e aplicação da manufatura aditiva para construção civil, focada na utilização de polímeros ABS e PLA, para criação de objetos ou módulos que possam ser estudados e testados em seus comportamentos mecânicos visando a otimização estrutural. Nesse sentido, as análises inicialmente arroladas se direcionam para levantamento de dados por meio de pesquisa bibliográfica onde a revisão de literatura permitiu uma visão sobre o que está sendo pesquisado e desenvolvido a respeito da análise estrutural de objetos produzidos com uso da impressora 3D. Ensaios destrutivos de corpos de prova, fabricados com os polímeros ABS e PLA, promovem o corpo da pesquisa, e a aferição do comportamento mecânico dos materiais faz com que essa análise possua embasamento para que seja, posteriormente, desenvolvida uma modelagem de componentes impressos com geometrias complexas. Este artigo é parte da fase inicial da pesquisa e vislumbra a otimização estrutural de peças, bem como a maior precisão de seu comportamento mecânico pósprodução.
\end{abstract}

Palavras-chave: Manufatura Aditiva, Impressão 3D, Performance Estrutural.

\begin{abstract}
The general objective of this master's research is based on the understanding, analysis and application of the additive manufacture for the construction industry, focused on the use of ABS and PLA polymers, for the creation of objects or modules which their mechanical behavior can be studied and tested in order to optimize structural. In this sense, the analyses initially listed are directed to data collection through a bibliographical research where the literature review allows an insight into what is being researched and developed regarding structural analysis of objects produced by using 3D printer. Destructive testing of specimens made with the ABS and PLA polymers promotes the body of the research and the measurement of the mechanical behavior of the materials causes this analysis to have a basis for the subsequent development of a modeling of printed components with complex geometries. This paper results from the initial phase of the research and envisages the structural optimization of parts, as well as the greater precision of its mechanical post-production behavior.
\end{abstract}

Keywords: Additive Manufacturing, 3D Printing, Structural Performance.

\section{INTRODUÇÃO}

Com o aumento da competitividade global, a diversificação dos requisitos do cliente, tendências de mercado, visam a integração dos processos de design, que desafiam cada vez mais a fabricação e o produto, a fim de reduzir o

SOUZA, E. H. F.; MARTINEZ, A. C. P.; TIBÚRCIO, T. M. S. Potencialidades da manufatura aditiva para construção civil. In: SIMPÓSIO BRASILEIRO DE QUALIDADE DO PROJETO NO AMBIENTE CONSTRUÍDO, 6., 2019, Uberlândia. Anais... Uberlândia: PPGAU/FAUeD/UFU, 2019. p 739-747. DOI https://doi.org/10.14393/sbqp19068. 
tempo de desenvolvimento e lidar com a crescente complexidade, sem comprometer a qualidade. A tecnologia de Manufatura Aditiva (MA), usualmente conhecida como impressão 3D, tem influenciado continuamente os ambientes de criação e de produção. Por muito tempo, arquitetos e engenheiros vêm buscando uma solução para impressão 3D no tocante relacionado a estruturas de grande escala (YUAN; CHEN; ZHANG, 2018).

Dentro do setor da MA, é possível gerar uma nova forma de construir, criando elementos sem limitações geométricas, conectando materiais já utilizados na construção tradicional com novos materiais, podendo criar conjuntos e formas, as quais podem possuir características mais relevantes que as tradicionais (complexa geometria, econômica e mais atrativa por exemplo). Estes novos elementos desenvolvidos podem ter também propriedades mecânicas que os tornem "especiais", sendo mais resistentes, mais leves, podendo ser transportados com maior facilidade e ou produzidos em locais com maior dificuldade de acesso, gerando uma forte inclinação para a inovação devido as mudanças nos domínios do design computacional e ferramentas avançadas de fabricação digital (NABONI; PAOLETI, 2015).

Segundo Crolla et al. (2017), atrelado a tudo isso, uma nova disposição de possibilidades para o design, para a Arquitetura, Engenharia e Construção (AEC) faz com que as formas possam ser traduzidas e otimizadas da melhor maneira possível, realizadas em formatos ainda desconhecidos devido à complexidade exigida. Experimentos interativos com modelos estruturais como um protótipo virtual, permitem prever o desempenho da estrutura por meio de simulação e melhorar de forma iterativa o desempenho do sistema.

O setor da construção civil normalmente apresenta algumas particularidades e complexidades. Yavuz e Sagiroglu (2016) afirmam que as construções são realizadas dentro dos limites e possibilidades de seu tempo, em termos de tipo, métodos de produção, qualidade, dimensões e técnicas de aplicação. Consequentemente, é conhecido que os edifícios se beneficiam, predominantemente, de materiais naturais e locais, que são usados na busca pelo menor esforço de produção.

Admitindo-se que as limitações dos processos de AEC têm contribuído para o cenário de atraso tecnológico e organizacional no setor da construção civil, pesquisas neste setor são necessárias para que sejam discutidas e testadas alternativas para a melhoria e inovação dos seus processos produtivos e construtivos.

\section{METODOLOGIA}

Esse artigo trata especificamente de resultados da fase inicial de uma pesquisa de mestrado, caracterizada por pesquisa documental e pela análise do comportamento mecânico de modelos impressos em 3D, avaliação de ensaios destrutivos de esforços de compressão axial e flexão e comportamento de impacto de tração, além do estudo comparativo da evolução da impressão. É uma pesquisa exploratória e qualitativa, onde foram analisados e comparados os três artigos: "Automatic method for fabricating a three-dimensional plastic model with photo-hardening polymer" por Kodama (1981), "Mesh Mould: robotically fabricated metal meshes as concrete formwork and reinforcement" por Hack et al. (2015) e "Evaluation of mechanical behavior of abs and pla polymers in 3D printing for structural 
performance simulation" por Martinez et al. (2017). A escolha destes três artigos deu-se devido a importância da transformação e evolução da MA ao longo dos últimos anos.

\section{RESULTADOS E DISCUSSÃO}

Para obter o conhecimento mais profundo sobre a manufatura aditiva e o comportamento mecânico de materiais poliméricos impressos em 3D, é necessário estudar as propriedades do material além de promover a conceituação do desenvolvimento desta tecnologia ao longo dos anos. Por este motivo, esta pesquisa retrata e analisa alguns modelos que foram desenvolvidos nos últimos anos (KODAMA 1981; HACK et al., 2015; MARTINEZ ef al., 2017; MASERA et al., 2017).

Visto através de um panorama mais abrangente, cerca de $40 \%$ do consumo mundial de energia e um terço das emissões mundiais de Gases de Efeito Estufa (GEE) são atribuíveis ao setor da construção. Pesquisas significativas e esforços legislativos foram feitos para reduzir as emissões de GEE durante as operações das edificações (emissões operacionais), enquanto as emissões produzidas durante a construção (emissões incorporadas) receberam muito menos atenção. Recentemente, o aumento do foco tem sido direcionado para as emissões incorporadas, uma vez que elas representam uma grande porcentagem do total de emissões de GEE no ciclo de vida do edifício. Esta percentagem tende a aumentar à medida que os edifícios se tornam mais eficientes em termos energéticos durante $O$ seu funcionamento (RIPPMANN et al., 2018).

Foi também enfatizado, recentemente, que a maior parte das emissões incorporadas se relaciona com a estrutura dos edifícios e em particular as lajes de piso e de telhado. Segundo os autores (DE WOLF, 2017; RIPPMANN et al., 2018) existem duas maneiras de reduzir as emissões de GEE incorporadas, a primeira alternativa é através da redução do volume de materiais utilizados e a segunda é utilizando materiais com baixo teor de carbono incorporado.

A MA representa uma forte alternativa para redução do volume de materiais utilizados nas construções e para utilização de materiais de menor impacto ambiental. Em relação aos materiais recicláveis e ecologicamente corretos, normalmente, apresentam menor resistência em comparação aos materiais comumente usados, no entanto, algumas alternativas estão surgindo para melhoria destes processos, como por exemplo o concreto baseado em Cimentos Ativados por Álcalis, baseados em resíduos reciclados adequado para ser usado em impressão 3D e processos alternativos de fabricação digital, mesmo que hoje possa ser considerado estruturalmente fraco (WANGLER et al., 2016).

Entretanto, novas pesquisas demonstram que a capacidade de carga suficiente pode vir através da geometria estrutural, em vez da resistência do material, fornecendo uma solução para esses materiais serem usados estruturalmente e com segurança (DE WOLF, 2017; RIPPMANN et al., 2018), abordando diretamente a segunda recomendação sobre a utilização de carbono de baixa incorporação materiais.

Segundo Yuan, Chen e Zhang (2018), o primeiro modelo sólido impresso foi projetado e executado por Hideo Kodama em 1981. Sua pesquisa foi realizada 
com o intuito de obter um novo método para fabricação automática de um modelo plástico tridimensional. Um modelo sólido foi fabricado expondo o polímero de foto-endurecimento líquido aos raios ultravioletas e empilhando as camadas solidificadas em seção transversal. A Figura 1 (A e B) em duas vistas, retrata o modelo impresso, por uma das três impressoras desenvolvidas pela pesquisa (KODAMA, 1981).
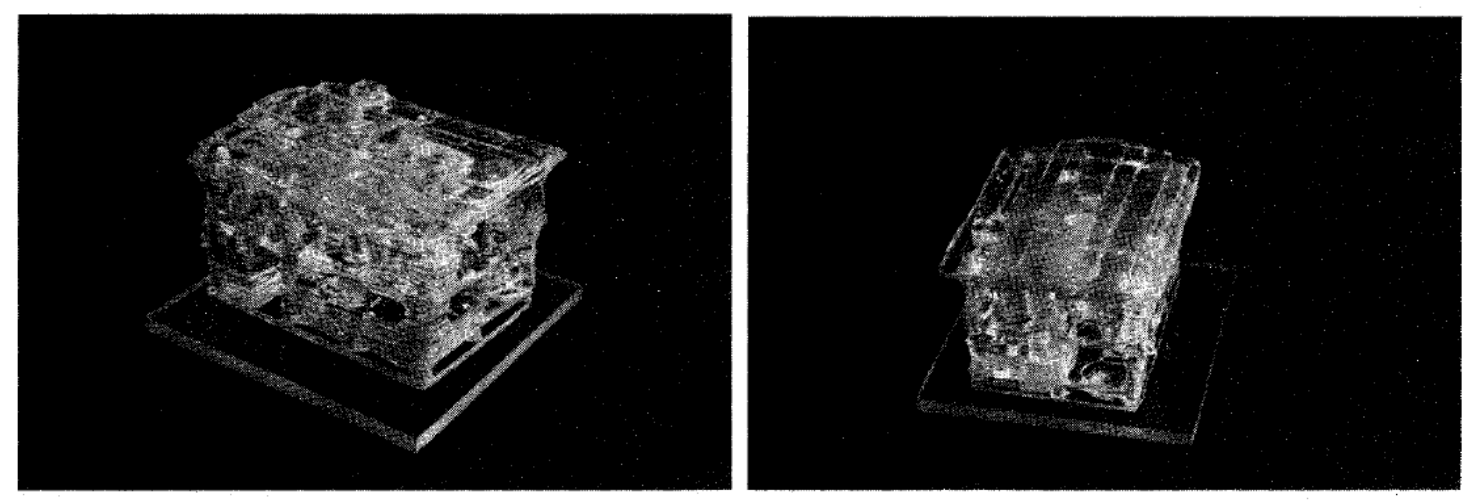

Figura 1 - (A) Modelo de casa impressa vista 01 e (B) Modelo de casa impressa vista 02 Fonte: KODAMA (1981)

De acordo com Kodama (1981), através de seu experimento verificou-se a possibilidade da produção de modelos sólidos de formas bastante complexas, os quais podem ser fabricados através desta técnica, permitindo que as estruturas internas possam ser fabricadas de uma só vez. Porém, seu modelo apresentou a deficiência da não fabricação de uma forma suspensa que não é suportada a partir de baixo. Alterando a sequência de solidificação das camadas, esses problemas podem ser evitados em algum grau, entretanto, esta é uma limitação básica desta técnica. Este problema já possui solução nos dias de hoje através da impressão composta, utilizando mais de um material, onde o material adjacente pode ser retirado através de soluções químicas. Mais conhecido por ter sido o primeiro modelo impresso, o protótipo de Kodama, abriu espaço para MA em todo cenário mundial.

A MA ainda está em processo de crescimento e amadurecimento, evoluindo em passo acelerado, sendo uma força motriz por trás de um novo paradigma industrial. Após mais de 25 anos de pesquisa, desenvolvimento e uso da impressão 3D em vários domínios industriais, como aeroespacial, automotivo, médico e recentemente no setor da AEC, continua a crescer com a adição de novas tecnologias, métodos e aplicações. É caracterizada como uma mudança da produção em massa para uma customização maciça nas fronteiras indefinidas entre bits e átomos, entre digital e material, entre projetar e produzir (LUCA, 2015; TAY et al., 2017).

O setor da MA vem crescendo e apresentando resultados positivos, em relação ao número de investimentos na área, apontando valores significativos no setor ao longo dos anos. De acordo com pesquisas realizadas por Luca (2015) e Masera et al. (2017), projeções em relação aos investimentos estão alcançando a casa dos bilhões, em relação ao mercado mundial estas projeções estão se multiplicando a medida que os anos se passam, gerando uma proporção próxima a duplicação dos investimentos a cada cinco anos, tudo isso graças aos avanços nas pesquisas, que geram novos materiais e novas formas de aplica-los. 
Graças a esta constante evolução da MA, diversas pesquisas foram realizadas, mostrando e comprovando a vasta aplicabilidade da impressão 3D. Por este motivo, este artigo apresenta e analisa de forma crítica modelos impressos, com variações de forma e de material, como é o caso do Mesh Mould, que explora a unificação de tipos de aplicações e formas diferentes em um único sistema de construção roboticamente fabricado (HACK et al., 2015).

Um robô industrial é usado para "tecer" espacialmente uma malha tridimensional, que atua como uma armadura durante o processo de despejo de concreto e é ativada como reforço após a cura do concreto. A pesquisa tem como objetivo desenvolver um processo de fabricação robótico in-situ totalmente automatizado (Figura 2 - (A), (B) e (C), respectivamente). A Figura 2 (A) mostra o braço robótico (KUKA) imprimindo a estrutura do módulo em $A B S$, a Figura 2 (B) é uma imagem aproximada do bico extrusor imprimindo o filamento para formação da estrutura e a Figura 2 (C) é a estrutura totalmente impressa e concretada da base até o meio do módulo, apresentando o resultado final da impressão com a concretagem.
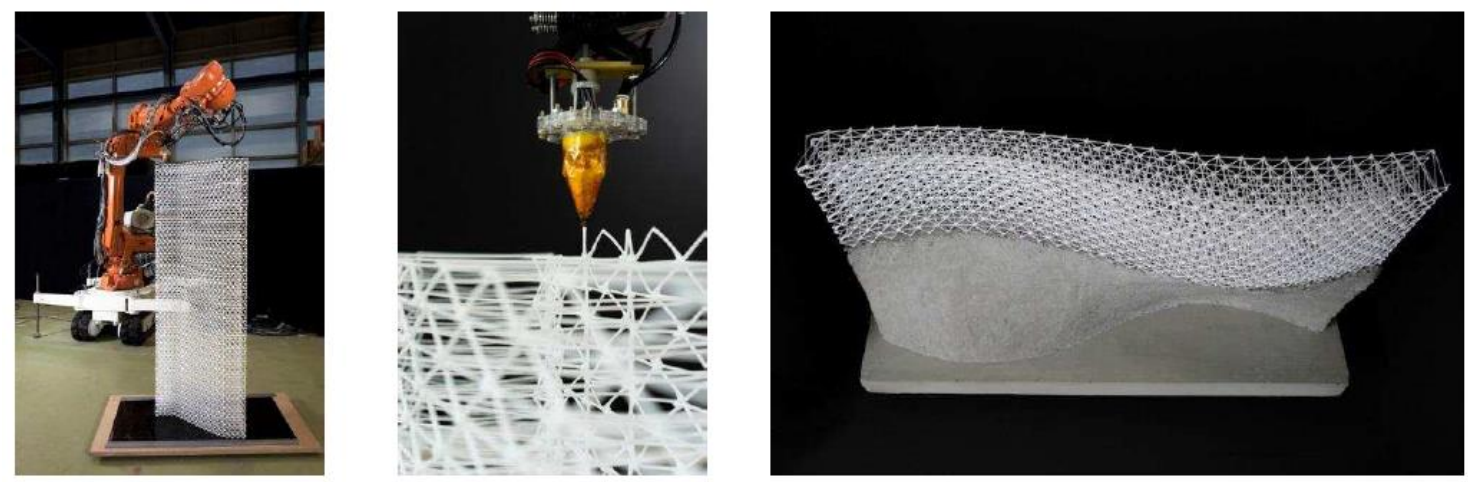

Figura 2 - (A) Impressão através do braço robótico,

(B) Bico extrusor no ato da impressão e (C) Sistema Mesh Mould de impressão 3D Fonte: HACK et al. (2015)

Na pesquisa realizada por Hack et al. (2015) o principal objetivo era a análise estrutural inicial, onde fosse possível identificar certas características particularmente bem-sucedidas da malha. Embora este teste tenha sido realizado por malhas finas de aço e ABS, sua geometria pode ser incorporada em outros materiais. Neste sentido, o processo de desenvolvimento a seguir descreve as orientações seguidas, através da determinação dos modelos desenvolvidos e testados assim como as características dos formatos das malhas, as geometrias internas e seu formato final, servindo de modelo e como comparação para outros testes. As principais orientações seguidas no desenvolvimento são demonstradas no Quadro 1.

Os elementos da pesquisa, se baseiam em duas partes, a primeira é composta pelo desenvolvimento e impressão 3D em ABS junto com a concretagem e a segunda parte é a fabricação de uma malha metálica através da dobra e soldagem, ambos as etapas foram realizadas através de braços robóticos em processo automatizado. A tipologia de malha e o processo de fabricação robótica, estão fortemente interligados e só podem ser vistos de maneira mutuamente dependente (HACK et al., 2015).

Aplicações na AEC estão ampliando o interesse da impressão 3D. No entanto, limitações da produção, dentre as quais inclui-se a performance estrutural, 
ainda impedem o uso significativo desta tecnologia. Neste sentido, a pesquisa de Martinez et al. (2017) apresenta parte da pesquisa de simulação e otimização estrutural e objetiva estudar o comportamento mecânico de polímeros $A B S$ e PLA, sob a forma de filamentos termoplásticos. Os resultados preliminares de avaliação do comportamento mecânico do ABS e PLA, caracterizada por ensaios destrutivos de esforços de compressão axial e flexão, impressos prioritariamente no modelo de impressora 3D Cubex Duo.

\begin{tabular}{|c|c|c|c|}
\hline \multicolumn{4}{|c|}{ Quadro 1 - Modelos, geometrias e interações } \\
\hline $\begin{array}{l}\text { MODELO } \\
\text { V1 }\end{array}$ & $\begin{array}{l}\text { Aumento da espacialidade através do } \\
\text { perímetro tetraédrico e da estrutura } \\
\text { interior diagonalmente espacial. }\end{array}$ & $\frac{\text { WWWWA If }}{X X X X}$ & $\begin{array}{r}\text { } \\
\square\end{array}$ \\
\hline $\begin{array}{l}\text { MODELO } \\
\text { V2 }\end{array}$ & $\begin{array}{l}\text { Aumento da espacialidade através do } \\
\text { perímetro tetraédrico e da estrutura } \\
\text { interior diagonalmente espacial. }\end{array}$ & $\frac{1 X X 1}{\mathrm{~V} 2}$ & $\begin{array}{r}W \\
\square \quad 20\end{array}$ \\
\hline $\begin{array}{l}\text { MODELO } \\
\text { V3 }\end{array}$ & $\begin{array}{l}\text { Número crescente dos fios que correm } \\
\text { horizontalmente }\end{array}$ & $\frac{\text { WWND: }}{\sqrt{\mathrm{N}}: \mathrm{V}}$ & 14111 \\
\hline $\begin{array}{l}\text { MODELO } \\
\text { V4 }\end{array}$ & $\begin{array}{c}\text { Montagem vertical como alternativa ao } \\
\text { layout horizontal }\end{array}$ & v4 & 1 \\
\hline $\begin{array}{l}\text { MODELO } \\
\text { V5 }\end{array}$ & $\begin{array}{c}\text { Variação da densidade das aberturas da } \\
\text { malha, desde o menor tamanho possível } \\
\text { até o maior tamanho de abertura capaz } \\
\text { de suportar o concreto }\end{array}$ & $\frac{W m}{\sqrt{\wedge \sqrt{\Lambda V}}}$ & $\begin{array}{l}\text { 11 } \\
1\end{array}$ \\
\hline
\end{tabular}

Fonte: HACK et al. (2015), adaptado pelos autores

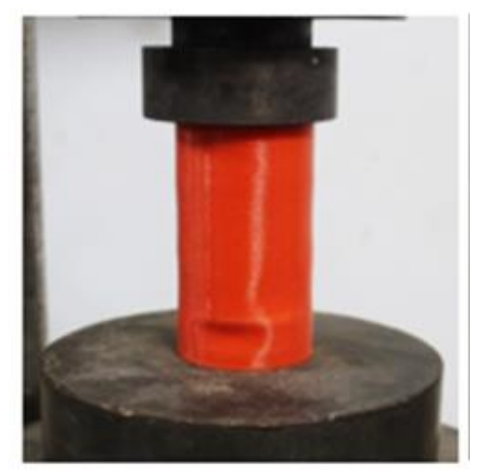

Figura 3 - Teste de compressão axial CP cilíndrico em PLA Fonte: MARTINEZ et al. (2017), adaptado pelos autores

A Figura 3 é um Corpo de Prova (CP) cilíndrico impresso em PLA com dimensões de $5,0 \mathrm{~cm}$ de diâmetro, $10,0 \mathrm{~cm}$ de altura e espessura de $0,5 \mathrm{~mm}$, submetido ao teste de compressão axial, onde apresenta sua deformação na parte inferior do CP, no qual obteve uma Tensão de 90,56 Mpa.

A Figura 4 é um Corpo de Prova (CP) cilíndrico impresso em ABS com dimensões de $5,0 \mathrm{~cm}$ de diâmetro, $10,0 \mathrm{~cm}$ de altura e espessura de 0,5 mm, submetido ao teste de compressão axial, onde apresenta sua deformação na parte inferior do CP, no qual obteve uma Tensão de 56,53 Mpa. 
A Figura 5 é um Corpo de Prova (CP) paralelepípedo impresso em ABS com dimensões de $2,0 \mathrm{~cm}$ de base, $3,0 \mathrm{~cm}$ de altura, $9,95 \mathrm{~cm}$ de largura e espessura de $0,5 \mathrm{~mm}$, submetido ao teste de flexão em três pontos, no qual obteve uma Tensão de 53,78 Mpa.

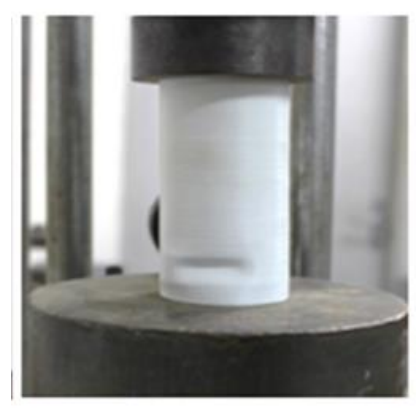

Figura 4 - Teste de compressão axial CP cilíndrico em ABS Fonte: MARTINEZ et al. (2017), adaptado pelos autores

Figura 5 - Teste de flexão em três pontos CP paralelepípedo em ABS Fonte: MARTINEZ et al. (2017), adaptado pelos autores

Segundo a pesquisa realizada por Martinez et al. (2017), onde diversos corpos de prova foram impressos e testados mecanicamente, num total de 18 modelos impressos nos filamentos PLA e ABS, compostos por um conjunto de 3 paralelepípedos $(3,0 \mathrm{~cm} \times 2,0 \mathrm{~cm} \times 15,0 \mathrm{~cm})$ e 4 cilindros nas dimensões de 2,5 $\mathrm{cm}$ de diâmetro e $5,0 \mathrm{~cm}$ de altura e $5,0 \mathrm{~cm}$ de diâmetro e $10,0 \mathrm{~cm}$ de altura. Um problema de destaque são as limitações das impressoras de baixo custo, que ainda são entraves para assegurar um rígido controle de resistência e qualidade uniforme dos modelos físicos. Além disso, sob as mesmas configurações de modelagem e controles de impressão (massa, densidade, tempo, temperatura, dentre outros), há também variações decorrentes das diferenças entre equipamentos (MARTINEZ et al., 2017).

Neste contexto, pode-se dizer que segundo os resultados de Martinez et al. (2017) o ABS possui melhor resistência a compressão com malha interna, porém, possui maior deformação dimensional. Já o PLA possui melhor resistência a compressão em corpos de prova maciços, porém, possui menor deformação dimensional, comprovando assim a melhoria mecânica dos protótipos produzidos através do seu material e geometria, promovendo avanços para sua utilização em modelos que exigem maior resistência. Os materiais poliméricos comumente processados já são estudados e avaliados, mas ainda não são impressos nas várias configurações de orientação.

\section{CONCLUSÕES}

Os três trabalhos apresentados por Kodama (1981), Hack et al. (2015) e Martinez et al. (2017), servem como exemplo para comprovar a evolução que 
a MA apresentou nos últimos anos, começando através de um modelo simples e muito pequeno, passando por diversas alterações a ponto de poder ser mesclado com outros materiais, servindo de estrutura, vedação e até mesmo como um módulo construtivo completo. A verificação do poder estrutural mecânico obtido através destes estudos comprova a vasta utilidade das impressões e mostra que ela ainda possui um amplo campo de estudo e aplicações. A partir do momento que se pode comprovar a sua boa resistência mecânica, através dos testes compressão e flexão, se torna possível uma total união entre a AEC tradicional/vernacular com tecnologias que já estão sendo testadas, como é o caso da MA, e também permite que novas tecnologias se conectem a este processo.

O desenvolvimento de novos materiais e o estudo das geometrias trazem constante mudança para o cenário da AEC. Estas mudanças podem provocar, com o passar dos anos, uma possível alteração no processo de construção, com o objetivo de prejudicar cada vez menos o meio ambiente e de fornecer aos seus usuários a qualidade necessária para moradia e estadia. A sociedade precisa de mudanças que gerem menos desgaste e degradação do espaço onde o ser humano vive e a MA vem como uma possibilidade para essa necessidade.

Através das análises realizadas após o estudo adentro dos artigos relacionados e todo conteúdo verificado, é possível dizer que na avaliação mecânica de materiais impressos, pode-se prever grande avanço nos próximos anos. 0 comportamento mecânico dos modelos avaliados propicia uma evolução eminente no setor da AEC. Aos olhos dos pesquisadores e profissionais da área, esse avanço reflete na qualidade dos modelos projetados e desenvolvidos, promovendo um melhoramento mecânico significativo que num futuro próximo pode constituir um novo modelo de construção com aplicações de materiais simples, possivelmente, reciclados e que apresentem boa resistência mecânica, segurança e qualidade do ambiente construído.

\section{AGRADECIMENTOS}

Ao Grupo de Pesquisa INOVA, ao Programa de Pós-Graduação em Arquitetura e Urbanismo e ao Departamento de Arquitetura e Urbanismo da UFV pelo apoio recebido.

\section{REFERÊNCIAS}

CROLLA, K.; WILLIAMS, N.; MUEHLBAUER, M.; BURRY, J. Smart Nodes Pavilion. Towards Custom-optimized Nodes Applications in Construction. In: CAADRIA, 22, 2017, Hong Kong. Proceedings... Hong Kong: CAADRIA, 2017. p. 467-477. ISBN 9789881902689.

DE WOLF, C. C. E. L. Low carbon pathways for structural design: embodied life cycle impacts of building structures. 2017. PhD Thesis. Massachusetts Institute of Technology (MIT).

HACK, N.; LAUER, W. V.; GRAMAZIO, F.; KOHLER, M. Mesh Mould: Robotically fabricated metal meshes as concrete formwork and reinforcement. In:

Proceedings of the 11th International Symposium on Ferrocement and 3rd ICTRC International Conference on Textile Reinforced Concrete, Aachen, Germany (pp. 7-10). 2015. 
LUCA, B. A Performative Approach To 3D Printed. Politecnico di Milano, Facoltà di Architettura e Società. Bachelor's in science of Architecture. 2015.

KODAMA, H. Automatic method for fabricating a three-dimensional plastic model with photo-hardening polymer. Review of scientific instruments, v. 52, n. 11 , p. 1770-1773, 1981.

MARTINEZ, A. C. P.; PEDROTI, L. G.; DE SOUZA, D. L.; DOS SANTOS, D. M.; MARTINS, M. A. D. Simulação de desempenho estrutural baseada na prototipagem rápida com impressão 3d. Blucher Design Proceedings, v. 3, n. 12, p. 333-339, 2017.

MASERA, G.; MUSCOGIURI, M.; BONGIOVANNI, A.; COLOMBO, M. Towards a new digital craft. Potential and limitations of 3D printing in architecture and construction. In: ISTeA. Re-shaping the construction industry. Maggioli Editore, 2017. p. 83-92.

NABONI, R.; PAOLETTI, I. Advanced customization in architectural design and construction. Springer International Publishing, 2015.

RIPPMANN, M.; LIEW, A.; VAN MELE, T.; BLOCK, P. Design, fabrication and testing of discrete 3D sand-printed floor prototypes. Materials Today Communications, v. 15, p. 254-259, 2018.

TAY, Y. W. D.; PANDA, B.; PAUL, S. C.; NOOR MOHAMED, N. A.; TAN, M. J.; LEONG, K. F. 3D printing trends in building and construction industry: a review. Virtual and Physical Prototyping, v. 12, n. 3, p. 261-276, 2017.

WANGLER, T.; LLORET, E.; REITER, L.; HACK, N.; GRAMAZIO, F.; KOHLER, M.; FLATT, R. Digital concrete: opportunities and challenges. RILEM Technical Letters, v. 1, p. 67-75, 2016.

YAVUZ, A. O.; SAĞIROĞLU,O. Reviewing the Bricks Used in The Traditional Architecture with The Shape Grammar Method. Gazi University Journal of Science, v. 29, n. 4, p. 741-749, 2016.

YUAN, P. F.; CHEN, Z.; ZHANG, L. FORM FINDING FOR 3D PRINTED PEDESTRIAN BRIDGES. Learning, Adapting and Prototyping, (CAADRIA) 2018, Volume 1, 225234. 2018. 